\title{
Effect of Inrush Current on Carbon Nanotube Synthesis from Xylene by Liquid-Phase Pulsed Arc Method Using Copper Electrodes
}

\author{
Takio Kizu* \\ Department of Electrical Engineering, Tokyo University of Science, \\ 1-3 Kagurazaka, Shinjuku, Tokyo 162-8601, Japan \\ Shinya Aikawa \\ International Center for Materials Nanoarchitectioncs, \\ National Institute for Materials Science, 1-1 Namiki, Tsukuba, Ibaraki 305-0044, Japan \\ Kentaro Takekoshi and Eiichi Nishikawa \\ Department of Electrical Engineering, Tokyo University of Science, \\ 1-3 Kagurazaka, Shinjuku, Tokyo 162-8601, Japan. \\ (Received 25 October 2012; Accepted 29 November 2012; Published 12 January 2013)
}

\begin{abstract}
We present the possibility of o-xylene as a carbon supplier for the synthesis of carbon nanotubes (CNTs) by the liquid-phase pulsed arc method using copper electrodes. Moreover, we investigate the effect of inrush current on the composition of the synthesized material. As inductance from an air-core coil increased, the weight ratio of copper particles tended to decrease dramatically. Moreover, with increasing inductance, the weight of the CNTs tended to increase. These results imply that control of discharge current is important for the synthesis of CNTs by the liquid-phase method. [DOI: 10.1380/ejssnt.2013.8]
\end{abstract}

Keywords: Carbon nanotubes; Arc discharge; Inrush current; Nanoparticles; Xylene

\section{INTRODUCTION}

Carbon nanotubes (CNTs) are distinctive in their mechanical, chemical, and physical properties; hence, they are considered to be promising next-generation carbon materials for nanowires, polymer composite materials, secondary cell electrode materials, field emission devices and biosensor elements [1].

Nanocarbon structures such as CNTs, carbon nanoonions, and carbon nanospheres are synthesized by arc discharge in vacuum, laser ablation, and chemical vapor deposition (CVD) $[1,2]$. Electric arc discharge in liquids is particularly simple and cost-effective because it does not require vacuum devices [3-6]. Although these factors are important for future application, it is difficult to control carbon impurities, especially graphite flakes from graphite electrodes that are generated by an arc method. In almost all research employing arc discharge in the liquid phase, the nanocarbons produced are composed of carbon atoms supplied from the graphite electrodes. For example, Sano et al. reported that carbon nano-onions could be synthesized by arc discharge in water [3]. Hsin et al. presented the first low-temperature, solution-phase production of CNTs using carbon plasma in an aqueous solution [4]. Muthakarn et al. reported that CNTs could be synthesized in toluene using graphite electrodes and suggested that the carbon sources of CNTs in this method are both liquid toluene and graphite electrodes [7].

Several groups have synthesized nanocarbon materials from liquid hydrocarbons. Shibata et al. reported that amorphous carbon particles could be produced in ultrasonically irradiated liquid benzene by the arc discharge method [8, 9]. According to a report by Okada et al., tube-like nanocarbons were formed by the arc discharge

*Corresponding author: nishilab@rs.kagu.tus.ac.jp method between nickel electrodes in toluene [10], although they appeared to have a low-crystallinity graphite-layer structure. We previously demonstrated the production of shorter and high-crystallinity CNTs using a sucrose solution as a carbon supplier [11]; however, electron microscopy revealed the formation of only a few nanotubes in the specimen, suggesting that the sample contained a high proportion of metal particles. Moreover, we recently found that discharge characteristics dependent on sucrose concentration play an important role in CNT synthesis by liquid-phase arc discharge [12]. This experiment determined that the number of electric sparks decreases with an increasing molar concentration of the sucrose solution. We postulated that the reduction in the number of electric sparks was attributed to the high liquid viscosity of the sucrose solution. In addition, it has been found that the number of electric sparks decreases with decreasing CNT production. These results suggest that the amount of carbon supplied cannot be increased by increasing the concentration of the sucrose solution. Thus, a low-viscosity carbon source is required to increase CNT production. Furthermore, it has been found that a large number of metal particles are present in the sample when the liquid-phase arc method is used. It is considered that the generation of metal particles is caused by the large transient current, which is also known as inrush current.

Herein, we present the possibility of o-xylene as a carbon supplier for the synthesis of CNTs by the liquid-phase arc discharge method. Furthermore, we investigate the effect of inrush current on the composition of the synthesized material.

\section{EXPERIMENTAL}

A schematic diagram of the experimental apparatus is shown in Fig. 1. Liquid xylene $(100 \mathrm{~mL})$ was prepared in a glass beaker, which was cooled in an ice water bath. 


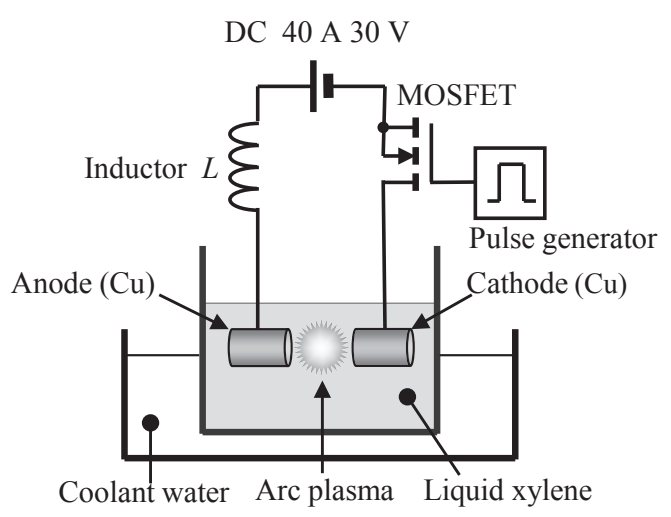

FIG. 1: Schematic diagram of the experiment by the arc discharge method in liquid xylene.

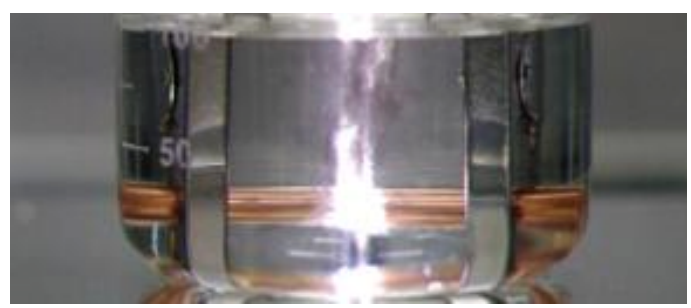

FIG. 2: Photograph of liquid-phase pulsed arc discharge using an air-core coil with an inductance of $330 \mu \mathrm{H}$. The pulse width and interval were set at 300 and $20 \mathrm{~ms}$, respectively.

Copper rods ( $5 \mathrm{~mm}$ in diameter, $99.9 \%$, Nilaco) were used as both an anode and a cathode. These electrodes were submerged in the glass beaker and then connected to a DC power supply, metal-oxide semiconductor field-effect transistor (MOSFET), pulse generator, and air-core inductor for inductance $L(L=0,10,134$, and $330 \mu \mathrm{H})$ in series with the anode. The pulse width and interval were set at 300 and $20 \mathrm{~ms}$. The voltage between the electrodes and the upper current limit of the power supply were set at $30 \mathrm{~V}$ and $40 \mathrm{~A}$, respectively. The interelectrode gap was maintained at less than $0.5 \mathrm{~mm}$. Discharge was operated for $15 \mathrm{~min}$, and discharge current waveforms were monitored by a digital oscilloscope (LeCroy WaveSurfer 24MXs) with a current probe (HIOKI 3274). Immediately after arc discharge as shown in Fig. 2, carbon soot was generated from the surface of the electrodes; thus, the liquid in the beaker turned black in $15 \mathrm{~min}$. The products in the beaker were filtered using a filter paper. The filtered residues were observed by a transmission electron microscope (TEM; Hitachi H-9500, operated at $200 \mathrm{kV}$ ). Thermogravimetric analysis (TGA) was performed by a thermogravimetric differential thermal analysis instrument (TG-DTA; SII Nano Technology EXSTAR 6200 ), and the products of this analysis were further analyzed by Raman spectroscopy (JASCO NRS-3000). The surface of the cathodes was observed by a scanning electron microscope (SEM; Carl Zeiss SUPRA 40, operated at $10 \mathrm{kV}$ ).

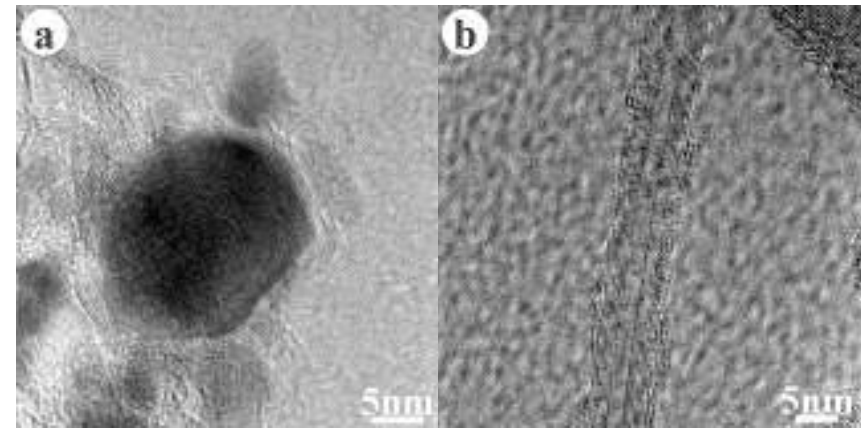

FIG. 3: Typical high-resolution TEM images of carbon nanoparticles by arc discharge in liquid using (a) no inductance, (b) with the air-core coil of an inductance of $330 \mu \mathrm{H}$.
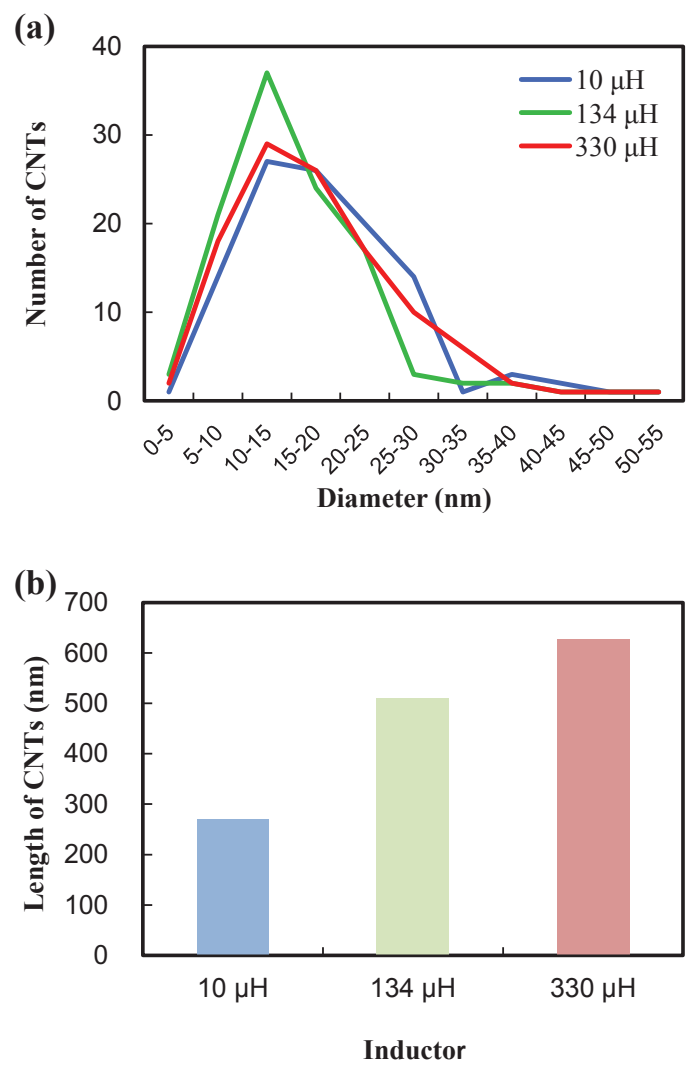

FIG. 4: Structural-size analysis of CNTs using TEM images. (a) Histogram of the diameter of CNTs and (b) Relationship between the average length of CNTs and inductance.

\section{RESULTS AND DISCUSSION}

TEM analysis of the as-grown sample revealed that the CNT purity was improved by use of the inductor ( $L=$ 10, 134, or $330 \mu \mathrm{H})$. On the other hand, a few CNTs were observed without use of the inductor. Figure 3(a) shows a typical TEM image of the carbon nanoparticles obtained without the inductor. In this image, we observed copper nanoparticles encapsulated in graphitic shells (i.e., carbon nanocapsules). The metal particles were approximately 5 to $30 \mathrm{~nm}$ in diameter, and the graphite layers numbered between 3 and 10. In addition, the graphite layer had high crystallinity. CNTs and carbon nano- 


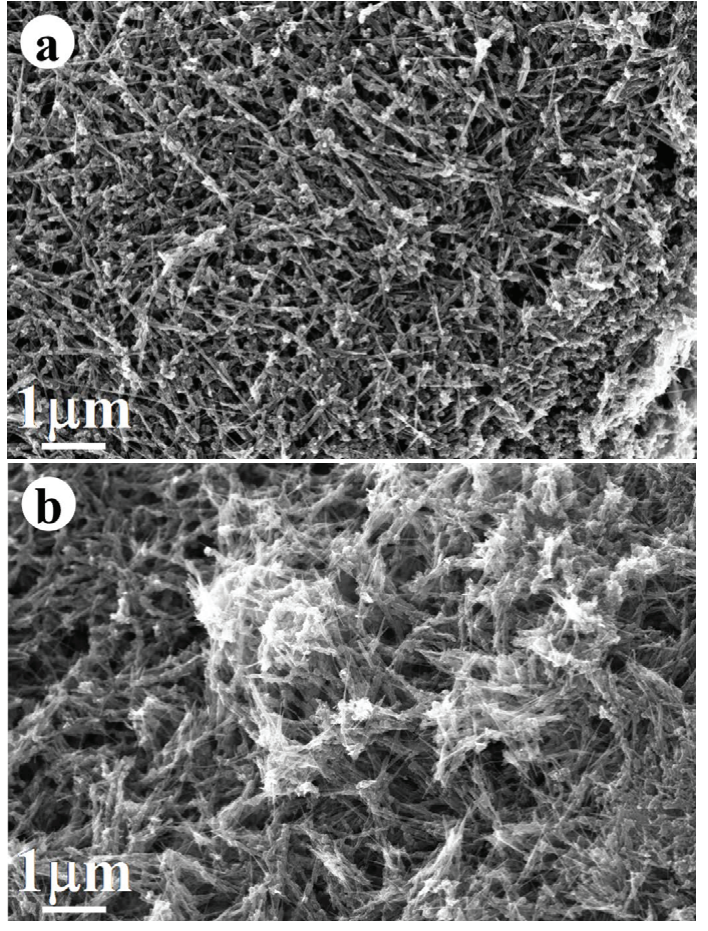

FIG. 5: SEM image of the copper cathode surface. CNT synthesis by arc discharge in liquid using copper electrodes.

onions were observed with TEM but were of low purity. Figure 3(b) shows a typical TEM image of multi-walled CNTs (MWCNTs) with high crystallinity when an inductance of $330 \mu \mathrm{H}$ was applied. Similarly, MWCNTs could be synthesized when an inductance of 10 or $134 \mu \mathrm{H}$ was applied. This TEM observation also confirmed that metal particles and spheroidal nanocarbons [10] were obtained as byproducts. Figure 4(a) shows a histogram of the diameter of the CNTs, which was estimated from the TEM images. According to the histogram, no significant difference was observed in the diameter distribution of CNTs in the presence of an inductor. Figure 4(b) shows the relationship between the average length of the CNTs and the inductance value. From the graph, it can be seen that the length of the CNTs tends to increase with an increase in the inductance value. This is believed to be caused by plasma stabilization associated with using an inductor. SEM observation results were not in agreement with the TEM analysis. Figure 5 shows typical SEM images of the cathode surface on which the products were deposited both without and with an inductance of $330 \mu \mathrm{H}$. Under both conditions, CNTs grew on the cathode surface after arc discharge. It is suspected that the entire amount of carbon was deposited on both electrode surfaces just before the end of the experiment. Thus, the quantity of carbon particles dispersed in the liquid o-xylene was increased.

Figure 6 shows TGA curves of each as-grown sample at a heating rate of $20^{\circ} \mathrm{C} / \mathrm{min}$ in dry air. The lines show a weight loss as a function of temperature from 30 to $1000^{\circ} \mathrm{C}$. With an inductance of 10,134 , or $330 \mu \mathrm{H}$, a weight loss was observed at around $250-450^{\circ} \mathrm{C}$, which corresponds to the combustion temperature of $\alpha-\mathrm{C}$ [13] and other carbon impurities. The range from 450 to $850^{\circ} \mathrm{C}$

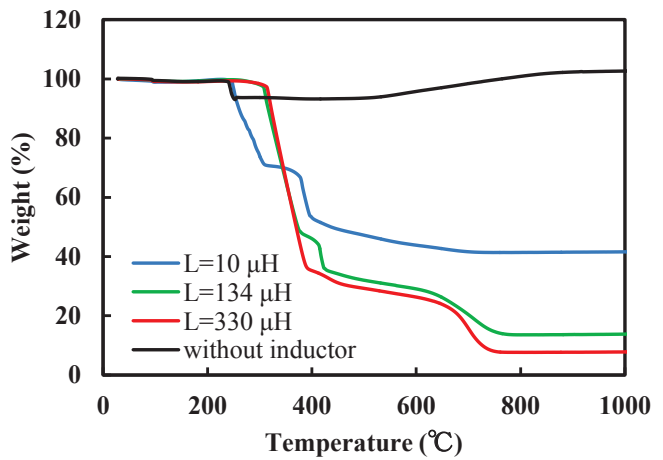

FIG. 6: TGA curve of the filtered residues. Black, blue, green, and red lines show TGA curve with inductance $L=0,10,34$, and $330 \mu \mathrm{H}$, respectively.

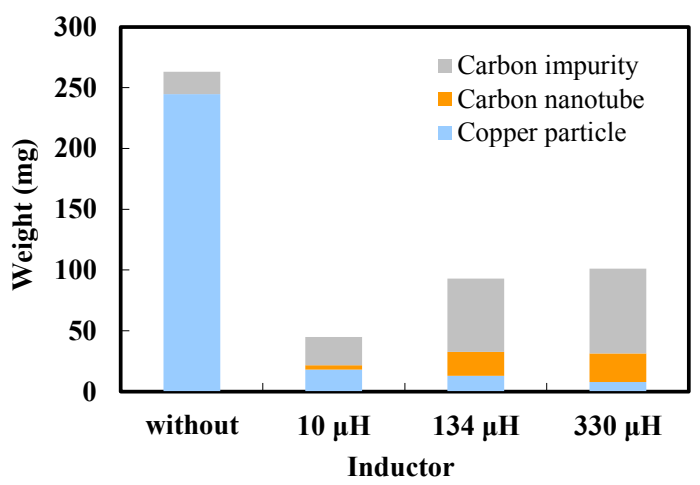

FIG. 7: Correlation between sample composition and inductance value.

corresponds to the combustion temperatures of MWCNTs. TGA data reveals that the ashed residuals are metal oxides. Without inductance, the sample weight increased from $250^{\circ} \mathrm{C}$, possibly the result of copper particle oxidation. Figure 7 shows the relationship between sample composition calculated from the TGA results and the inductance of the air-core coil. With increases in inductance, the weight ratio of metal particles tends to dramatically decrease. Moreover, with increasing inductance, the weight of CNTs and carbon impurities tends to increase. These results are consistent with the TEM observation results. A typical Raman spectrum of the sample after oxidation from 100 to $600^{\circ} \mathrm{C}$ in dry air using TG-DTA is shown in Fig. 8. For the oxidation sample with an inductance of $330 \mu \mathrm{H}$, G-band $\left(1590 \mathrm{~cm}^{-1}\right)$ and D-band $\left(1350 \mathrm{~cm}^{-1}\right)$ peaks are clearly seen. These peaks originate. from the MWCNTs as shown in Fig. 3(b). Without inductance, no peaks originating from CNTs can be observed in this spectrum. Meanwhile, we suppose that the spectrum shown in Fig. 8(a) is due to copper oxide particles.

Figure 9 shows typical current and voltage waveforms (shown as the lower black and upper red lines, respectively) monitored by an oscilloscope. The relationship between the inductance and average inrush current is shown in Fig. 10. We estimate the average of the inrush current from the peak current of a one-shot pulse discharge. The error bars shown in the figure are standard deviations of 


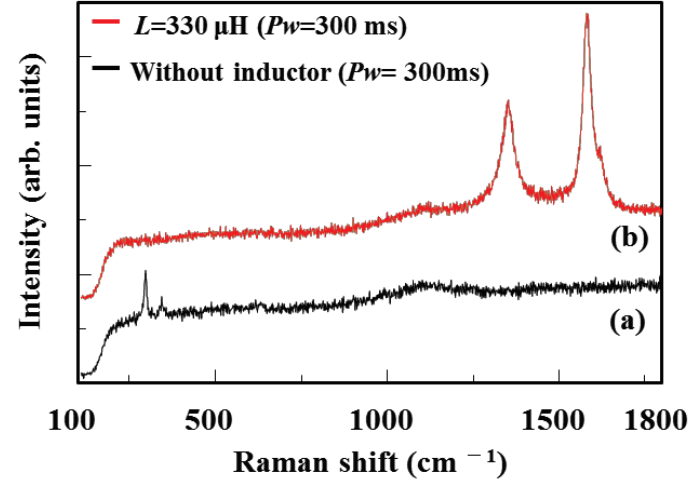

FIG. 8: Raman spectrum of the products after TG analysis. (a) Without inductor, (b) with air-core coil of $330 \mu \mathrm{H}$.
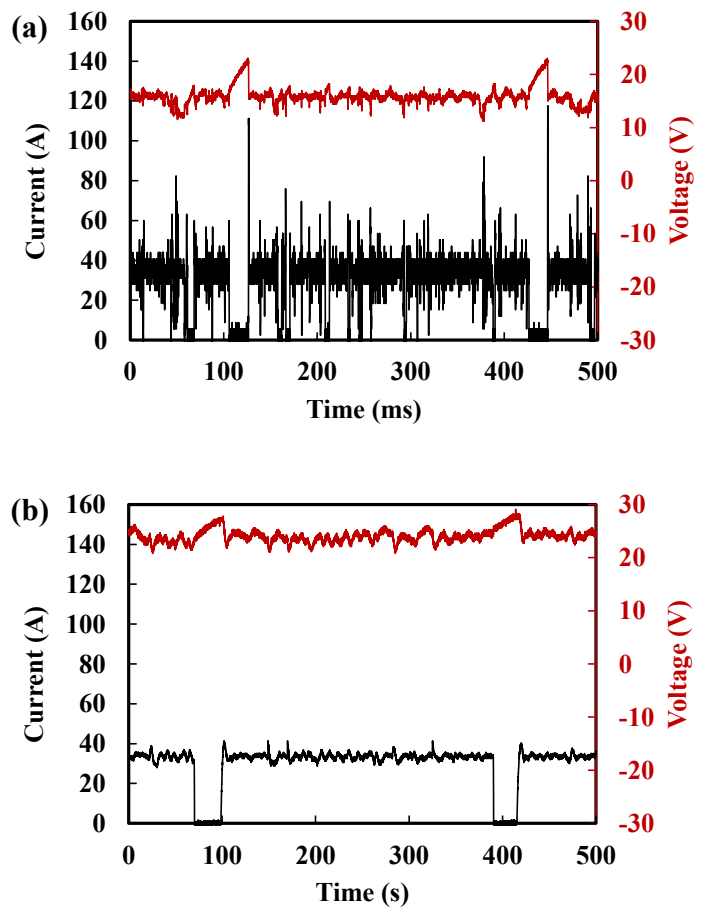

FIG. 9: Typical current and voltage waveforms of arc discharge in liquid. (a) Without inductor, (b) with an air-core coil of $330 \mu \mathrm{H}$.

the inrush current. With increases in the inductance of the coil connected to the discharge circuit, the inrush current decreases. In addition, as the inductance increases, the inrush current volatility tends to decrease. From this result, it was observed that arc discharge can be stabilized by use of an inductor. A stable arc discharge is believed to play an important role in CNT synthesis by the liquidphase method.

Generally, for arc discharge between metal electrodes in vacuum, many electrons are emitted from the cathode immediately after the initiation of discharge. As an emitted electron collides with the anode, the temperature at the point of collision is increased, and a metal ion is emitted from the cathode. In this study, as soon as a copper ion is emitted from the copper electrodes, carbon particles deposit on both electrodes. When the inrush current is

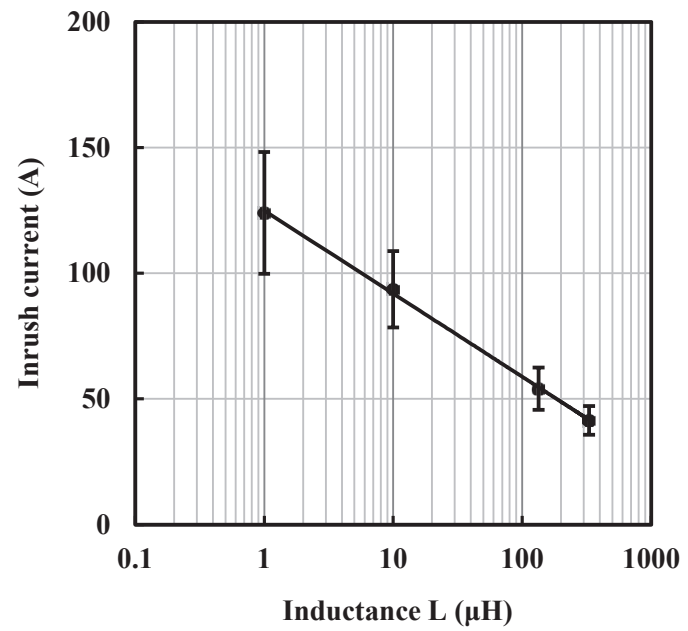

FIG. 10: Relationship between inductance and inrush current. Error bars are standard deviations of the inrush current.

high, the rate of copper ion emission is also high, thereby generating the graphite capsule containing copper particulates [Fig. 3(a)]. As a result, it is believed that the deposition rate is high for copper particulates that originate from the compound under no inductance. On the other hand, when the inrush current is low, it is suspected that the evaporation of copper is controlled and that the rate of carbon deposition on an electrode increases, approaching the case of electric discharge between carbon electrodes. As previously reported by Sano et al., the temperature of the arc plasma in water is approximately $4000 \mathrm{~K}$ [14]. Similarly, it is believed that the temperature of the liquid-phase plasma is approximately $3000 \mathrm{~K}$ because the boiling point of a copper electrode is $2835 \mathrm{~K}$. Consequently, we deduced that the temperature of the arc plasma at the electrode in this method is approximately 3000-4000 K. Carbon clusters and $\mathrm{C}^{+}$ions were generated from o-xylene as a result of decomposition by the high-temperature plasma. The carbon vapor cooled rapidly at the gas-liquid interface and then deposited on the surface of the electrode. As a result, the CNT growth mechanism of liquid-phase method became similar to case of arc discharge method using carbon electrodes. Thus it is concluded that the growth mode of the CNTs generated in our study follows a vapor-liquid-solid mechanism [15].

\section{CONCLUSIONS}

We have demonstrated the synthesis of highcrystallinity CNTs by arc discharge in liquid o-xylene using copper electrodes. We also investigated the relationship between the inrush current and the purity of synthesized CNTs. The results indicate that as inductance increases, the weight of the CNTs tends to increase. It was also found that the discharge current can be stabilized using an inductor. Therefore, it is important to note that controlling the discharge current waveform plays a significant role in the synthesis of CNTs by this method. 
[1] A. Jorio, G. Dresselhaus, and M. S. Dresselhaus, Carbon Nanotubes: Advanced Topics in the Synthesis, Structure, Properties and Applications. (Springer, Berlin, 2008).

[2] S. Iijima, Nature 354, 56 (1991).

[3] N. Sano, H. Wang, M. Chhowalla, I. Alexandrou, and G. A. J. Amaratunga, Nature 414, 506 (2001).

[4] Y. L. Hsin, K. C. Hwang, F.-R. Chen, and J.-J. Kai, Adv. Mater. 13, 830 (2001).

[5] H. Lange, M. Sioda, A. Huczko, Y. Q. Zhu, H. W. Kroto, and D. R. M. Walton, Carbon 41, 1617 (2003).

[6] K. Imasaka, Y. Kanatake, Y. Ohshiro, J. Suehiro, and M. Hara, Thin Solid Films 506, 250 (2006).

[7] P. Muthakarn, N. Sano, T. Charinpanitkul, W. Tanthapanichakoon, and T. Kanki, J. Phys. Chem. B 110, 18299 (2006).

[8] E. Shibata, R. Sergiienko, H. Suwa, and T. Nakamura, Carbon 42, 885 (2004).
[9] R. Sergiienko, E. Shibata, H. Suwa, T. Nakamura, Z. Akase, Y. Murakami, and D. Shindo, Ultrasonics Sonochemistry 13, 6 (2006).

[10] T. Okada, T. Kaneko, and R. Hatakeyama, Thin Solid Films 515, 4262 (2007).

[11] S. Aikawa, T. Kizu, E. Nishikawa, and T. Kioka, Chem. Lett. 36, 1426 (2007).

[12] S. Aikawa, T. Kizu, K. Kamei, and E. Nishikawa, e-J. Surf. Sci. Nanotech. 9, 215 (2011).

[13] M. S. Dresselhaus and P. C. Eklund, Adv. Phys. 49, 705 (2000).

[14] N. Sano, H. Wang, I. Alexandrou, M. Chhowalla, K. B. K. Teo, G. A. J. Amaratunga, and K. Iimura, J. Appl. Phys. 92, 2783 (2002).

[15] Y. Saito, T. Yoshikawa, M. Inagaki, M. Tomita, and T. Hayashi, Chem. Phys. Lett. 204, 277 (1993). 